УДК 004.942

\title{
Self-assembly of Three Nanoparticles in Structures with a Predetermined Geometry Controlled by Quasiresonant Laser Field
}

\author{
Viktoria S. Kornienko \\ Aleksey S. Tsipotan* \\ Vitaliy V. Slabko ${ }^{\dagger}$ \\ Siberian Federal University \\ Svobodny, 79, Krasnoyarsk, 660041
}

Russia

Received 16.03.2018, received in revised form 10.06.2018, accepted 29.08.2018

\begin{abstract}
In the paper a dynamic model of self-assembly of nanoparticles under laser field is proposed for research the possibility of a step-by-step assembly of a structure consisting of three particles with different form. The possibility of forming such structures with a predetermined geometry from a preformed pair of particles is considered.
\end{abstract}

Keywords: dipole, laser field, self-assembly of nanostructures, colloidal crystals. DOI: 10.17516/1997-1397-2018-11-6-702-711.

\section{Introduction and preliminaries}

Recently, the problems of the electric dipole particles interaction with each other in an external field attract the interest of researchers in various fields of science and technology [1]. In particular, a theoretical study of the electric dipoles properties, their interaction and behaviour in an external electric field lead to the creation of a theory of dielectrics and the explanation of a number of physical effects [2,3]. A simulation of the interaction of a large number of atmospheric dipoles with charged particles makes it possible to explain thunderstorm and other atmospheric effects [4].

In recent decades, nanostructures with unique properties that differ from the properties of a bulk sample and are dependent on both composition and shape are actively being studied. Therefore, the question of developing an universal method for the formation of nanostructures is actively discussed by researchers. The least expensive method of obtaining colloidal crystals (highly ordered structures consisting of nanoparticles) that does not require local physical influence on the system is based on the ability of nanoparticles to self-organize in the process of random Brownian collisions in real disperse systems [5]. However, with such an approach it is impossible to control the processes of predetermined nanostructures formation. One of the possible solutions to this problem is the physical effects on ensembles of nanoparticles that allow the formation of complex nanostructures without additional surface modifications [6], however, it is not always possible to achieve the selectivity of the self-assembly process. Note that the modelling

\footnotetext{
*acipotan@sfu-kras.ru

†vslbko49@mail.ru

(C) Siberian Federal University. All rights reserved
} 
of self-assembly processes is widely covered in the modern literature, as it gives a good qualitative and quantitative description of real physical processes and allows obtaining preliminary parameters for carrying out experiments. Thus, a model for describing the self-assembly of $\mathrm{CdTe}$ nanoparticles into helical structures due to light-induced charges was presented in [7], and the processes of nanoparticles self-organization under the action of a non-resonant electromagnetic field with frequencies in a wide range were considered in [8].

It was also shown in [9] that the interaction of resonant nanosized particles in the field of laser radiation makes it possible to form predefined structures, since the energy of the induced dipoledipole interaction is sufficient to overcome the barrier preventing spontaneous aggregation (which makes it possible to form a stable structure), and their geometry depends on the wavelength and the polarization of the external field. The previous studies (both theoretical and experimental) [10-12] on the formation of colloidal quantum dots pairs in the field of laser radiation showed the possibility of realizing this method. The further formation of more complex structures (three or more particles) can be realized in the course of a step-by-step process, when a third particle is added to an already formed pair of particles, by selecting the wavelength of the external field.

In this paper a dynamic model of self-assembly of nanoparticles under a laser radiation field is proposed with the aim of investigating the possibility of forming a three-particle structure with different shapes, as well as selecting the necessary parameters of the medium for conducting a physical experiment and predicting its results.

\section{Model for the formation of nanostructures}

The method of forming structures with a predetermined geometry as a result of self-assembly of resonant nanoparticles in the field of laser radiation was proposed in [11-13]. The essence of the method is as follows. The effect of laser radiation on microobjects causes their polarization, which is the cause of the interaction of the particle both with the external field, which makes it possible to form structures with dimensions on the order of the radiation wavelength, and with other particles. In the latter case it is possible to form structures with dimensions much smaller than the wavelength of the laser radiation causing this polarization. The existence of optical resonances of the particle leads to an increase in the interparticle interaction. This can be the basis for the selective formation of various structures with a predetermined position of the particles in the aggregate, since the energy of the interparticle interaction in the laser radiation field depends on its frequency, the resonance frequencies of the particles and the orientation of their group relative to the plane of polarization. A potential well is formed in the spectral and angular dependence of the energy of the interparticle interaction corresponding to the given configuration of the group. If the depth of this well exceeds the energy of the barrier [14], which prevents spontaneous aggregation, then a self-organized fusion of the considered group into a nanostructure is possible, with a predetermined configuration determined by two independent parameters: the frequency and the polarization of the laser radiation.

For the description the particles self-organization in the laser radiation field, we use the model described in [15].

\section{Formulation of the problem}

Let us consider an ensemble consisting of $N$ particles with mass $m_{j}$, radius $R_{j}$ and a resonant frequency $\omega_{j}^{0}$. The particles in the ensemble interact with each other through the dipole moments 
induced by the external light field. For each $j$-th dipole particle at each instant of time $t$, we define the following quantities:

$$
\begin{aligned}
& \vec{r}_{j}=\left(r_{j}^{x}, r_{j}^{y}, r_{j}^{z}\right)^{T} \text { is the position of the center of mass of the dipole particle; } \\
& \vec{V}_{j}=\left(V_{j}^{x}, V_{j}^{y}, V_{j}^{z}\right)^{T} \text { is the vector of translational velocity of the center of mass; } \\
& \vec{d}_{j}=\left(d_{j}^{x}, d_{j}^{y}, d_{j}^{z}\right)^{T} \text { is the vector of the dipole moment. }
\end{aligned}
$$

At the modeling of the structure formation process in 100 one-type calculations, we had been finding the probability that within a time $T \leqslant 10$ ns the center-to-center distance between the particles becomes $\left|\vec{r}_{i j}\right|=2 R_{j}$, and also the dependence of this probability on the particle orientation relative to the plane of the laser radiation polarization and its frequency.

We assume that the dimensions of the ensemble are much smaller than the wavelength of the incident radiation, which makes it possible to regard the external light field $\vec{E}$ throughout the ensemble as homogeneous and not to take into account the delay [13]. Then the vector of the electric component of the light wave field can be represented as a harmonic function with a frequency of oscillations $\omega^{r}$, which depends only on time, $\vec{E}=(1 / 2) \vec{E}_{0} \exp \left(i \omega^{r} t\right)+$ c.c.

Considering a local field near a $j$-th particle, in addition to the external field, it is necessary to take into account the contribution of all the remaining particles by summing the fields of the dipoles induced on them. Then the dipole moment vector induced on the $j$-th particle is:

$$
\vec{d}_{j}=\chi_{0 j}\left(\vec{E}+\sum_{i \neq j} \vec{E}_{k}\right)
$$

where $\chi_{0 j}$ is the linear polarizability of an isolated $j$-th particle, $\vec{E}_{k}$ is the field induced by the $k$-th particle of the ensemble at the location of the $j$-th particle:

$$
\vec{E}_{k}=\frac{1}{4 \pi \varepsilon_{0}} \frac{3\left(\vec{d}_{k}, \vec{r}_{j k}\right) \vec{r}_{j k}-\vec{d}_{k}\left|\vec{r}_{j k}\right|^{2}}{\left|\vec{r}_{j k}\right|^{5}},
$$

where $\vec{r}_{j k}=\vec{r}_{j}-\vec{r}_{k}, \varepsilon_{0}=\left(4 \pi c^{2}\right)^{-1} \cdot 10^{-9} \mathrm{C}^{2} /\left(\mathrm{N} \cdot \mathrm{m}^{2}\right)$ is the electric constant. Here $c$ is the speed of light.

In accordance with [16] and using (2) we obtain an expression describing the energy of the $j$-th dipole.

$$
W_{j}^{d}=\frac{1}{4 \pi \varepsilon_{0}} \sum_{i \neq j} \frac{\left(\vec{d}_{j}, \vec{d}_{k}\right)\left|\vec{r}_{j k}\right|^{2}-3\left(\vec{d}_{j}, \vec{r}_{j k}\right)\left(\vec{d}_{k}, \vec{r}_{j k}\right)}{\left|\vec{r}_{j k}\right|^{5}} .
$$

To describe the dipole polarizability of particles, we will use a two-level model [5]. Within the framework of this model, in the simplest case of a single resonance at a frequency $\omega^{r}$ characterized by the dipole moment of the transition $\left|d_{12}\right|^{2}$ and the excitation relaxation rate (homogeneous width), the dipole polarizability of the particle of the neighborhood $\omega^{r}$ can be described by the following expression:

$$
\chi_{0}=\frac{\left|d_{12}\right|^{2}}{\hbar(\Omega+i)}
$$

where $\Omega=\omega^{r}-\omega$ is the detuning from the resonance, $\hbar$ is the Planck constant. 


\subsection{Motion equations}

The translational motion of the $j$-th particle of the ensemble is described by the system of Langevin equations.

$$
\left\{\begin{array}{l}
d \vec{r}_{j} / d t=\vec{V}_{j}, \\
m_{j}\left(d \vec{V}_{j} / d t\right)=\vec{F}_{j}+\vec{F}_{j}^{f}+\vec{F}_{j}^{c},
\end{array}\right.
$$

where $\vec{F}_{j}=-\nabla\left(W_{j}^{v}+W_{j}^{e}+W_{j}^{d}\right), \vec{F}_{j}^{f}$ is the viscous friction force, $\vec{F}_{j}^{c}$ is the stochastic hydrodynamic force.

The pair interaction includes the Van der Waals energy and the electrostatic repulsion used in this work. To describe the Van der Waals interaction, we use the following expression [5]

$$
W_{j}^{v}=-\frac{A_{H}}{6} \sum_{i \neq j}\left(\frac{2 R_{j}^{2}}{h_{j k}^{2}+4 R_{j} h_{j k}}+\frac{2 R_{j}^{2}}{h_{j k}^{2}+4 R_{j} h_{j k}+2 R_{j}^{2}}+\ln \frac{h_{j k}^{2}+4 R_{j} h_{j k}}{h_{j k}^{2}+4 R_{j} h_{j k}+2 R_{j}^{2}}\right)
$$

where $A_{H}$ is the effective Hamaker constant, $R_{j}$ is the particle radius, $h_{j k}=\left|\vec{r}_{j k}\right|-R_{j}-R_{k}$ is the interparticle gap.

The potential energy $W_{j}^{e}$ of the Coulomb interaction of overlapping double electric layers for a $j$-th spherical particle of radius $R_{j}$ is given by the well-known expression [16]:

$$
W_{j}^{e}=2 \pi \varepsilon \varepsilon_{0} R_{j} \phi_{0}^{2} \sum_{i \neq j} \ln \left[1+\exp \left(-h_{j k} k_{0}\right)\right]
$$

where $k_{0}$ is the screening constant; $\varepsilon$ is dielectric permittivity of the environment; $\phi_{0}$ is the potential at the boundary of the Helmholtz layer.

It is assumed that the parameters of the medium are chosen so that the force of electrostatic repulsion exceeds the Van der Waals forces, but the effect of laser radiation could lead to aggregation only in resonant structures.

The viscous friction force, which is the main factor of dissipation of the kinetic energy, for spherical particles of radius $R_{j}$ is determined by the Stokes formula

$$
\vec{F}_{j}^{f}=-6 \pi \eta R_{j} \vec{V}_{j}
$$

here $\eta$ is the dynamic viscosity of the medium.

The interaction of particles with the environment with a fluctuating density leads to a random change in the trajectory of their motion, which should compensate the effect of the frictional force and preserve the mobility of the dispersed phase. To realize the Brownian motion, we assume that the random force $\vec{F}_{j}^{c}$ has a Gaussian distribution. During the time step $\Delta t$, the particle is constantly subjected to the action of the random force $\left(\vec{F}_{j}^{c}\right)^{k},(k=x, y, z)$. Before each step of integration, the magnitude of the projections of the random force on the coordinate axis is chosen from the Gaussian distribution with zero mean and deviation.

$$
\delta^{2}=\frac{12 \pi \eta R_{j} k_{b} T}{\Delta t}
$$

here $k_{b}$ is the Boltzmann constant, and $T$ is the temperature of the medium.

Taking into account all the forces acting on the particles in the quasiresonance radiation field, it is possible to model the process with the help of Brownian dynamics and to estimate the necessary parameters of the medium and the external field. In the general case, the integration of system (5) can be performed only numerically. 


\subsection{Model parameters}

According to the estimates given in $[9,13,15-17]$ as the initial data, we will choose the following parameters:

$$
\begin{gathered}
T=300 \mathrm{~K}, \eta=0.8902 \mathrm{mPa}, R_{j}=1.5 \mathrm{~nm},\left|d_{12}\right|^{2}=1.91 \cdot 10^{-43} \mathrm{~J} \cdot \mathrm{m}^{3}, \\
\lambda_{j}^{0}=525 \mathrm{~nm}, \phi_{\delta}=2.34 \mathrm{mV}, \Delta \lambda=3 \mathrm{~nm}, m_{j}=2.12 \cdot 10^{-23} \mathrm{~kg}, A_{H}=50 k_{b} T .
\end{gathered}
$$

The intensity of the laser field $I$ is defined as $I=10^{6} \mathrm{~W} / \mathrm{cm}^{2}$. At the initial instant of time $t=t_{0}$ the translational velocity is set equal to zero. The minimum distance $d_{m i n}$ between the centers of mass of dipole particles is defined as two lengths of the particle radius $d_{\text {min }}=2 R_{j}$. To find the numerical solution of the ODE system consisting of equations (5), we used the second-order method from the family of explicit Runge-Kutta methods [18].

\subsection{Numerical experiment}

The particle motion was calculated in a spherical cell in which $N$ particles are distributed at the initial time. The radius of the cell is set to about $20 \mathrm{~nm}$. At each step of the iteration, for each particle, whether isolated or part of the aggregate, the equations of motion (5) are solved in accordance with the model parameters described in paragraph 3.2 .

The numerical method is implemented with the step $\Delta t=0.01 \mathrm{~ns}$. The random force $\vec{F}_{j}^{c}$, as was shown in 3.1, has a Gaussian distribution. For its numerical realization, using a random number generator, a random, uniformly distributed quantity was assigned, and then the Box-Muller transformation was reduced to the Gaussian distribution in accordance with the distribution parameters described in 3.1. The probability of assembling particles into a structure was estimated from a sample consisting of 100 one-type calculations. The deviation from the mean value did not exceed $10 \%$.

\section{A pair of fixed particles}

Earlier, the possibility of forming pairs with various combinations of metallic and semiconductor particles in a quasiresonant laser field was demonstrated in [4, 10-12]. Therefore, in this paper we consider the possibility of step-by-step formation of complex structures of $N=3$ semiconductor nanoparticles, the parameters of which are defined in 3.2. We assume that particles 1 and 2 are fixed and their orientation in space is realized by an additional light field. To stabilize the orientation, it is necessary to choose non-resonant radiation with an intensity of about $I=10^{6} \mathrm{~W} / \mathrm{cm}^{2}$. Thus, the problem consists in the numerical calculation of the interaction energy between the third particle and particles 1 and 2 as a function of the wavelength and the polarization of the external field, which would allow us to determine the values of these parameters for the formation of a potential well with maximum depth. This, in turn, means that aggregation of particles is possible with the formation of stable structures of nanometer scale, in which the position of the particles is pre-determined. The position of the particles at the initial instant of time is described in Fig. 1. The distance between a pair of particles was chosen equal to $\left|\vec{r}_{12}\right|=R_{1}+R_{2}$, the distance between the second and third particles $\left|\vec{r}_{23}\right|$ are 20,16 or 10 $\mathrm{nm}$, the angle between the lines $\left|\vec{r}_{12}\right|$ and $\left|\vec{r}_{23}\right| \theta$ can be chosen arbitrarily.

The dependence of the dipole-dipole interaction energy, divided to the thermal energy $k T$ at the room temperature, of three nanoparticles (with a fixed pair) on the wavelength of the external field and the polarization angle is shown in Fig. 2, $\vec{r}_{23}=16 \mathrm{~nm}: \theta=0^{\circ}$ (left), $\theta=120^{\circ}$ (right). 


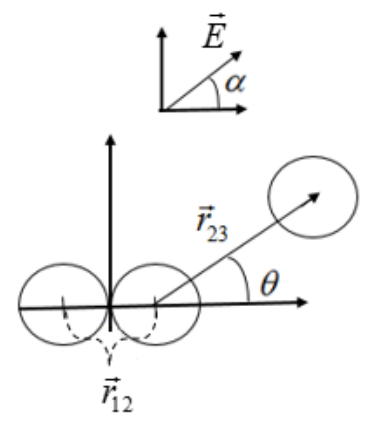

Fig. 1. Initial particles position

As can be seen from Fig. 2, the direction of polarization of the external field, as well as its wavelength and the location of the third particle, strongly influence the character of the energy spectrum.
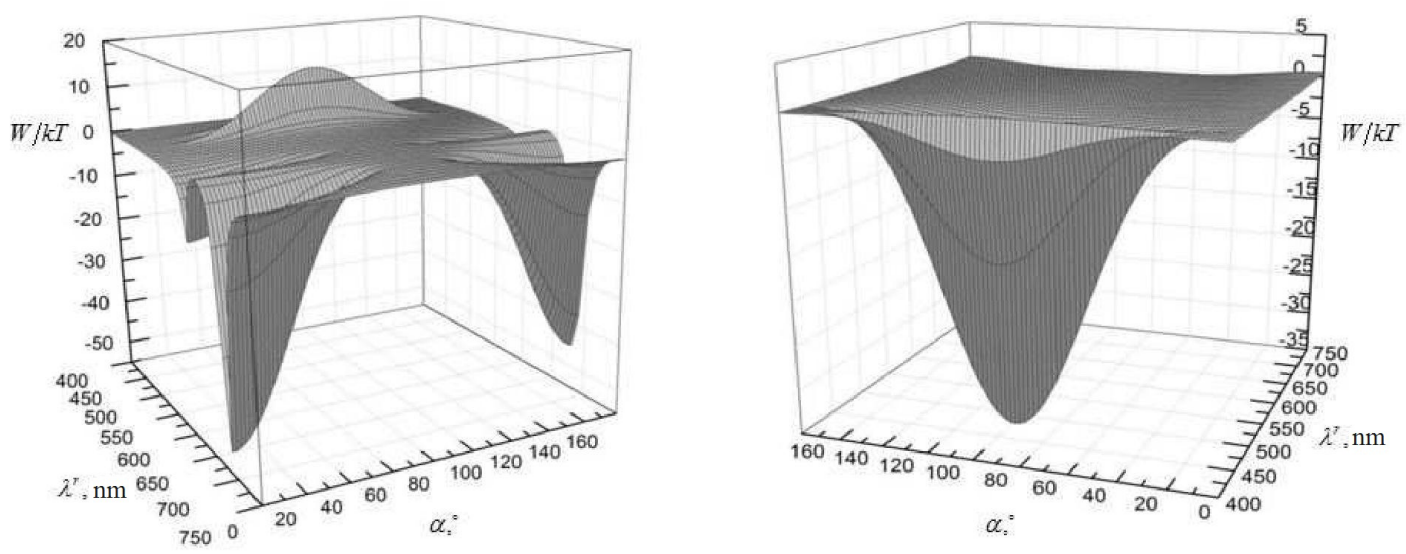

Fig. 2. Dependence of the energy of the dipole-dipole interaction of three nanoparticles (with a fixed pair) on the polarization angle $\alpha$ and the external field length $\lambda^{r}, \vec{r}_{23}=16 \mathrm{~nm}, \quad \theta=0^{\circ}$ (left), $\quad \theta=120^{\circ}$ (right)

For the angle $\theta=0^{\circ}$ for $\alpha=0^{\circ}$ and $\alpha=180^{\circ}$ (Fig. 2 on the left), a potential well in the interaction energy of particles of a depth of the order of $-50 \mathrm{kT}$, which means the formation of a stable aggregate of a pair of particles. The spectral width of the well is about $20 \mathrm{~nm}$. A different picture can be seen for the angle $\theta=120^{\circ}$, where a potential well of the order of $-33 k T$ is observed for $\alpha=90^{\circ}$ (Fig. 2 on the right). In this case, the potential well is shifted to the blue region by $450 \mathrm{~nm}$.

As the distance between the fixed pair and the third free particle decreases, the detuning from the resonance increases, and the resonance shifts to the red (blue) region for the first and second cases, respectively (Fig. 3).

Thus, the wavelengths $\lambda^{r}=690 \mathrm{~nm}$ and $\lambda^{r}=450 \mathrm{~nm}$ correspond to the maximum probability of stable nanostructures formation for a fixed pair. The chosen arrangement of particles relative to the plane of polarization corresponds to the maximum probability of formation of the structure. 


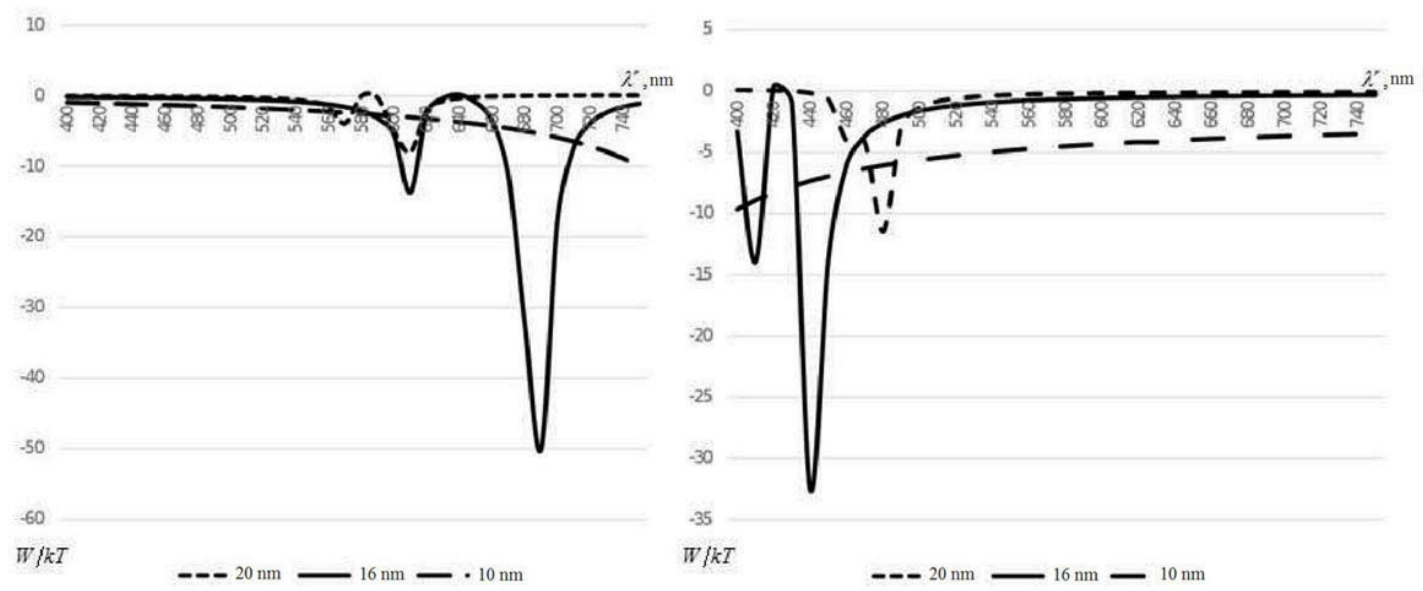

Fig. 3. The energy of the dipole-dipole interaction as a function of the distance between particles at $\theta=0^{\circ}, \alpha=0^{\circ}$ (left), $\theta=120^{\circ}, \alpha=90^{\circ}$ (right)

Obviously, in the course of Brownian motion, the particles can be arranged in a random position relative to each other and the external laser field.

We placed the third particle on a circle with a center in particle 2 and a radius of $16 \mathrm{~nm}$. The wavelength of the external field is $\lambda^{r}=690 \mathrm{~nm}$. Then for the angle $\alpha$ we obtained the following probability of stable nanostructures formation in the form of a line and a pyramid for a time not exceeding $10 \mathrm{~ns}$ (Fig. 4).
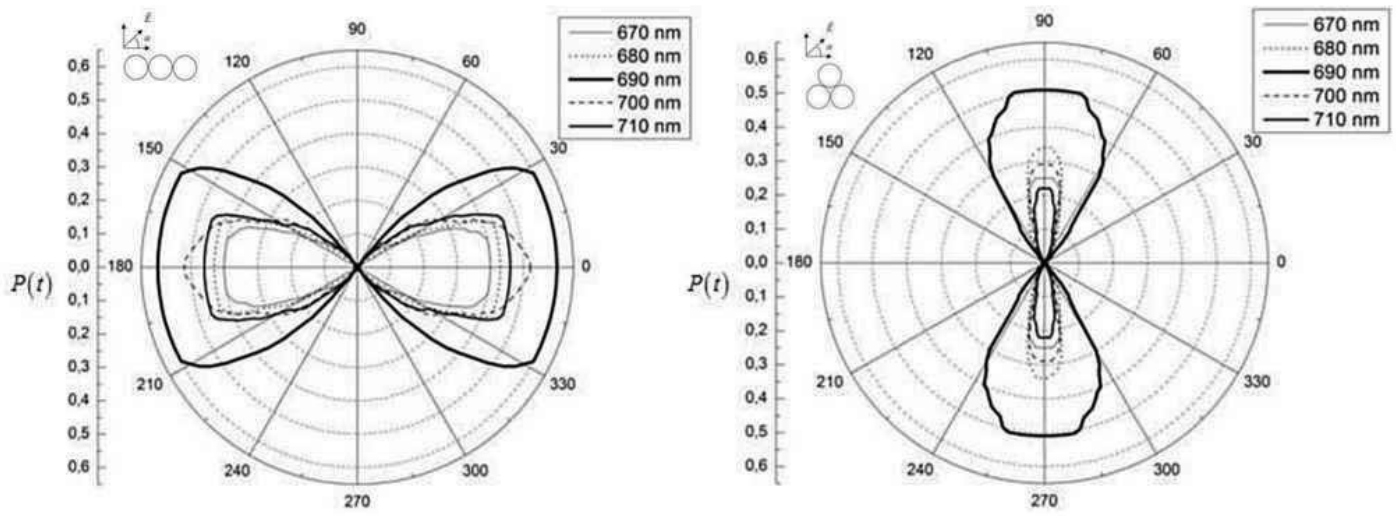

Fig. 4. The probability of aggregation of a triple of particles in a line (on the left) and a pyramid (on the right), depending on their mutual orientation at $\lambda^{r}=670-710 \mathrm{~nm}$

The average aggregation time of the three particles in the line is $5.72 \mathrm{~ns}$ and $5.36 \mathrm{~ns}$ for the pyramid. As can be seen from Fig. 4, the probabilities of forming both a line and a pyramid in one pulse reach $60 \%$ and $50 \%$, respectively. In this case, the angles of the direction of the external field polarization corresponding to the maximum of nanoparticles assembly are $0^{\circ}$ for the line and $90^{\circ}$ for the pyramid, which allows selectively to form the necessary structure. Similar calculations for the wavelength of the external field $\lambda^{r}=450 \mathrm{~nm}$ are shown in Fig. 5 .

Thus, for a fixed pair of particles at an external-field wavelength of $690 \mathrm{~nm}$, the most likely 
structure is a line, and the probability peak is reached at $\alpha=0^{\circ}$. Accordingly, for the wavelength of the external field of $450 \mathrm{~nm}$, the most probable structure is a pyramid with a probability peak for $\alpha=90^{\circ}$. Moreover, since the regions in which the formation of both structures are possible intersect, the region in which the process can be considered selective is narrowed.
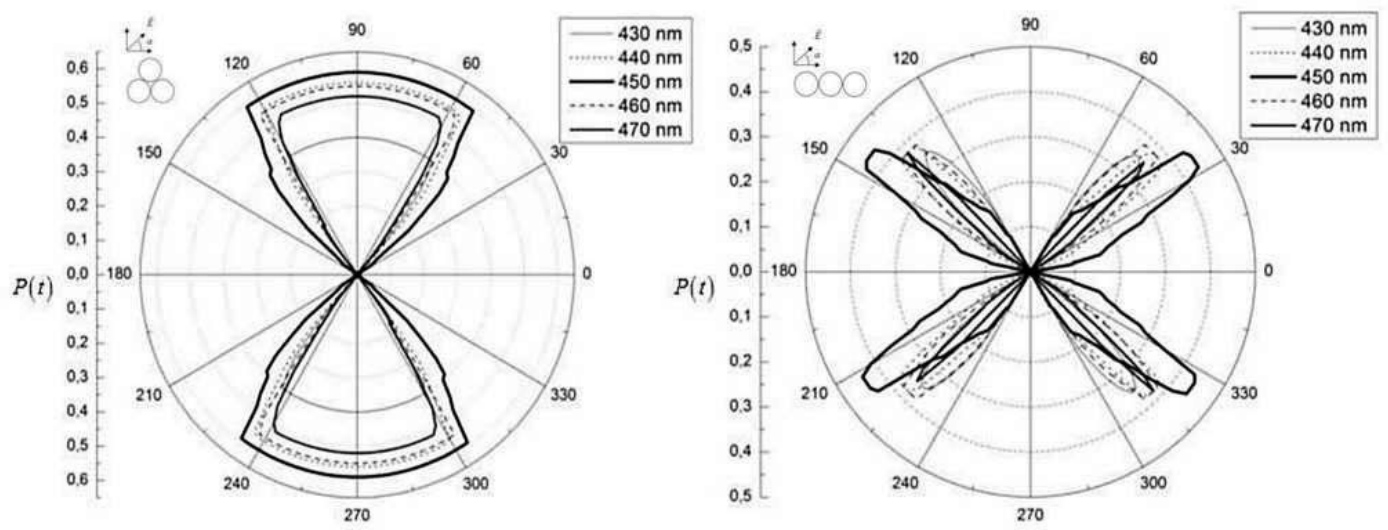

Fig. 5. The probability of aggregation of the triplet of particles into the pyramid (left) and the line (right), depending on their mutual orientation at $\lambda^{r}=430-470 \mathrm{~nm}$

As was shown earlier $[9,13,15]$, the selectivity of the structure formation depends not only on the angle, but also on the wavelength of the external field. So we considered how the detuning from the resonant wavelength corresponding to effective assembly affects the probability of forming the required three-particle structure (Figs. 4 and 5).

It can be seen from the figures that when the deviation from resonance decreases, the probability of assembly decreases, and the spread of the angle at which the probability of aggregation is maximal narrows. At the same time, because of the asymmetry of the potential well, deviation from resonance by the same step in the red or blue region gives different results. Also increases the assembly time in the structure to an average of $8.3 \mathrm{~ns}$ per line and $9.2 \mathrm{~ns}$ per pyramid, respectively. A similar picture is observed for the resonance in the red spectrum of the waves. Fig. 5 presents calculations of the probability of aggregation of particles for wavelengths $430-470$ $\mathrm{nm}$ into structures most probable for the resonance wavelength $\lambda^{r}=450 \mathrm{~nm}$.

The assembly time for deviation from the resonant wavelength also increases from $4.4 \mathrm{~ns}$ to $7.3 \mathrm{~ns}$ for the pyramid and from $4.7 \mathrm{~ns}$ to $8 \mathrm{~ns}$ on average.

The considered model was tested in experiment [9] for the formation of a pair of identical semiconductor CdTe particles in an aqueous colloidal solution using a frequency-tunable pulsed laser with a pulse duration of the order of tens of nanoseconds, providing an intensity of $10^{6} \mathrm{~W} / \mathrm{cm}^{2}$. The error between the experimental results and the model approximation was not more than $7 \%$.

\section{Conclusion}

In this paper, we demonstrated the fundamental possibility of forming a three-particle structure in the course of a step-by-step assembly in the field of laser radiation due to the induced dipole-dipole interaction. It was shown that it is possible to attach the third at a given angle to the already formed pair of particles fixed in space, choosing the wavelength and orientation of the electric field vector of the external field. The probability of formation of the structure 
reached $60 \%$ with an appropriate choice of field parameters, and the formation time was about $10 \mathrm{~ns}$. The parameters of the medium and the fields used in the experiment correspond to the parameters of the commercial parametric light generator, which indicates the possibility of realizing this method in practice, and the high probability of assembling the structure in $10 \mathrm{~ns}$ (during one pulse) allows us to assume that on average the structure can not be formed more than 2 pulses. A pulse duration of the order of $10 \mathrm{~ns}$ and a frequency of their repetition of the order of $10 \mathrm{~Hz}$ allow the medium to come to a state of thermodynamic equilibrium after each pulse, which will avoid the destruction of the structure due to prolonged laser action.

$V$.V. Slabko is grateful for the support from the Ministry of Education and Science of the Russian Federation (Grant no. 3.6341.2017/VU).

\section{References}

[1] E.Purcell, D.Morin, Electricity and Magnetism, Cambridge University Press, UK, 2013.

[2] R.P.Feynman, R.B.Leighton, M.Sands, The Feynman Lectures on Physics, London, Addison-Wesley Publishing Company, vol. 2, 1964.

[3] Ch.Kittel, Introduction to solid state physics, Wiley, 1996.

[4] V.V.Shaidurov, V.S.Kornienko, A.V.Vyatkin, The mathematical modeling of grouping the dipole water clusters, 3rd International Conference on Analysis and Applied Mathematics, Almaty, AIP Conference Proceedings, 1759(2016), no. 020004.

[5] S.V.Karpov, I.L.Isaev, A.P.Gavrilyuk, General principles of the crystallization of nanostructured disperse systems, Colloid Journal, 71(2009), no. 3, 313-328.

[6] J.Gong, G.Li, Z.Tang, Self-assembly of noble metal nanocrystals: Fabrication, optical property, and application, Nano Today, $\mathbf{7}(2012)$, no. 6, 564-585.

[7] S.Srivastava, A.Santos, K.Critchley, Light-Controlled Self-Assembly of Semiconductor Nanoparticles into Twisted Ribbons, Science, 327(2010), no. 5971, 1355-1359.

[8] J.Park, W.Lu, Self-assembly of nanoparticles into heterogeneous structures with gradient material properties, Physical Review E, 83(2011), no. 3, 031402.

[9] A.S.Tsipotan, Self-assembly of nanoparticles controlled by resonant laser light, Diss. for the degree of candidate of physical and mathematical sciences, 2015 (in Russian).

[10] V.V.Slabko, A.S.Tsipotan, A.S.Aleksandrovsky, Resonant light-controlled self-assembly of ordered nanostructures. Photonics and nanostructures. Fundamental and Applications, 10(2012), 636-643.

[11] V.V.Slabko, A.S.Tsipotan, A.S.Aleksandrovsky, E.A.Slyusareva, Dynamics of self-organized aggregation of resonant nanoparticles in a laser field, Springer-Verlag Berlin Heidelberg, Applied Physics B: Laser and Optics, 2014, 271-278.

[12] V.V.Slabko, A.S.Tsipotan, A.S.Aleksandrovsky, Self-organised aggregation of a pair of practices with different resonant frequencies and electric dipole moments of transitions, controlled by an external quasiresonant field, Quantum Electronic, 43(2013), no. 5, 458-462. 
[13] V.V.Slabko, G.G.Khachatryan, A.S.Alexandrovsky, Self-organized aggregation of small metal particles controlled by an external light field, Pisma $v$ Zh. eksper. Teoret. Fiz., 84(2006), 360-364 (in Russian).

[14] S.V.Karpov, V.V.Slabko, Optical and photophysical properties of fractal-structured metal sols, Novosibirsk, 2003 (in Russian).

[15] A.S.Tsipotan, A.S.Aleksandrovsky, N.E.Lyamkina, V.V.Slabko, Controlled self-assembly of nanoparticles under resonant laser light, News of higher educational institutions. Physics, 56(2013), no. 2/2, 314-319 (in Russian).

[16] N.Jacob, Intermolecular and surface forces, Academic Press, USA, 1992.

[17] A.S.Tsipotan, V.V.Slabko, N.V.Abuzova, Self-Assembly of nanopracticles Controlled by Resonant Laser Light, Journal of Siberian Federal University. Mathematics and Physics, 8(2015), no. 1, 109-122.

[18] N.S.Bahvalov, Numerical methods, Moscow, Nauka, 1975 (in Russian).

\section{Самосборка структур, состоящих из трех наночастиц, с заранее заданной геометрией под действием квазирезонансного лазерного излучения}

Виктория С. Корниенко Алексей С. Ципотан

Виталий В. Слабко

Сибирский федеральный университет Свободный, 79, Красноярск, 660041

Россия

В рамках данной работы предложена динамическая модель самосборки наночастич в поле лазерного излучения с иелью исследования возможности поэтапной сборки структуры, состоящей из трех частии, с различной формой. Рассмотрен процесс формирования таких структур с заданной геометрией в варианте присоединения к заранее сбормированной паре частии, третъей частицы.

Ключевые слова: диполь, лазерное поле, самосборка наноструктур, коллоидные кристаллы. 